\title{
Bias Amplification and Bias Unmasking
}

\author{
Joel A. Middleton \\ Department of Political Science, University of California, Berkeley, Barrows Hall, Berkeley, CA \\ 94720, USA \\ e-mail: joel.middleton@berkeley.edu (corresponding author) \\ Marc A. Scott \\ Department of Humanities and Social Sciences in the Professions, 246 Greene St., \\ New York University, Steinhardt, NY 10003, USA \\ e-mail:marc.scott@nyu.edu \\ Ronli Diakow \\ New York City Department of Education, 131 Livingston Street, Brooklyn, NY 11201, USA \\ e-mail:rdiakow@gmail.com \\ Jennifer L. Hill \\ Department of Humanities and Social Sciences in the Professions, 246 Greene St., \\ New York University, Steinhardt, NY 10003, USA \\ e-mail: jennifer.hill@nyu.edu \\ Edited by Prof. R. Michael Alvarez
}

\begin{abstract}
In the analysis of causal effects in non-experimental studies, conditioning on observable covariates is one way to try to reduce unobserved confounder bias. However, a developing literature has shown that conditioning on certain covariates may increase bias, and the mechanisms underlying this phenomenon have not been fully explored. We add to the literature on bias-increasing covariates by first introducing a way to decompose omitted variable bias into three constituent parts: bias due to an unobserved confounder, bias due to excluding observed covariates, and bias due to amplification. This leads to two important findings. Although instruments have been the primary focus of the bias amplification literature to date, we identify the fact that the popular approach of adding group fixed effects can lead to bias amplification as well. This is an important finding because many practitioners think that fixed effects are a convenient way to account for any and all group-level confounding and are at worst harmless. The second finding introduces the concept of bias unmasking and shows how it can be even more insidious than bias amplification in some cases. After introducing these new results analytically, we use constructed observational placebo studies to illustrate bias amplification and bias unmasking with real data. Finally, we propose a way to add bias decomposition information to graphical displays for sensitivity analysis to help practitioners think through the potential for bias amplification and bias unmasking in actual applications.
\end{abstract}

\section{Introduction}

In the analysis of causal effects in non-experimental studies, the key assumption necessary for unbiased estimation is that all confounders (pretreatment variables that are part of the data-generating mechanism for both treatment assignment and outcome) have been measured. In the social science literature, this assumption is often referred to as "selection on observables" (Heckman and Robb 1985, 1986), "conditional independence" (Lechner 2001), or "ignorability" (Rubin 1978), and it is well known that violation of this assumption leads to biased inference. However, it is typically implausible

Authors' note: For replication files, see Middleton (2016). Supplementary Materials for this article are available on the Political Analysis Web site.

(C) The Author 2016. Published by Oxford University Press on behalf of the Society for Political Methodology.

All rights reserved. For Permissions, please email: journals.permissions@oup.com 
to believe that we have measured all confounders, raising the question as to which of the available covariates should be adjusted for (conditioned upon) in practice.

Advice in the extant literature on which variables to condition on is contradictory. One recommendation has been that conditioning on more, rather than fewer, available (pretreatment) covariates is the best way to minimize bias associated with unobserved sources of heterogeneity (Rosenbaum 2002; Rubin 2002). Another recommendation says that those variables that are related to the treatment assignment mechanism should be included in the analysis (D'Agostino 1998). Still other advice is to choose covariates based on their relationship to the outcome, rather than to the treatment (Austin, Grootendorst, and Anderson 2007; Hill 2007; Brookhart et al. 2010). There is even some controversy within the controversy: in political science, Clarke (2005) contends that researchers are encouraged to include as many covariates as possible, without regard to the potential for increased bias; relatedly, in most intermediate econometrics texts, the choice between fixed and random effects is framed as a bias versus efficiency trade-off, with the approach that includes more predictors, fixed effects, described as unbiased (under assumptions that we contend are overly optimistic; see, e.g., Greene [2000]).

There are, however, two notable classes of covariates that most agree should be excluded from the set of conditioning covariates. These are bias inducers and bias amplifiers. Bias inducers include posttreatment variables such as mediators and colliders (Pearl 2000; Schisterman, Cole, and Platt 2009; Cole et al. 2010), and a particular group of pretreatment covariates (pretreatment colliders that lead to $M$-bias or butterfly-bias; Pearl 2009; Sjlander 2009; Ding and Miratrix 2014). Such bias inducers may not be troublesome in practice, however, either because they can be identified for exclusion, as is sometimes the case for posttreatment variables, or because the bias they induce tends to be small (Greenland 2002; Liu et al. 2012; Ding and Miratrix 2014).

Bias amplifiers have received recent attention as variables that should be excluded from the conditioning set (Bhattacharya and Vogt 2007; Wooldridge 2009; Pearl 2010; Myers et al. 2011; Pearl 2011; Wyss et al. 2014). These covariates cannot induce bias where there is none, but they increase bias by modifying bias that is due to an unobserved confounder. Instruments, variables that only affect the outcome through their impact on the treatment, are the canonical example of a bias-amplifying covariate. Conditioning on an instrument can hurt but can never help. On the one hand, this may seem like a trivial concern because it is unclear under what circumstances a researcher would be unaware that a variable was a true instrument for their treatment variable. However, even imperfect instruments can amplify bias (cf. Pearl 2010) and, as we will show below, even non-instruments can amplify bias.

The purpose of this article is to clarify the relationship between predictor inclusion and bias through a novel decomposition that serves to unify separate strands in the literature, which purport to make recommendations for variable inclusion or exclusion. We then discuss specific conditions in which fixed effects for groups can be detrimental (bias increasing), even though they are largely regarded as addressing a form of omitted variable bias benignly. We show, using a placebo test in a constructed observational study, how this decomposition and an enhanced graphical display devised for sensitivity analysis can better inform variable selection in the context of bias from an unobserved confounder.

The outline of the article is as follows. In Section 2, we derive our main results, showing how omitted variable bias may be decomposed into several constituent parts: bias attributable to the unobserved confounder, bias due to omitting observed covariates, and bias due to amplification. These decompositions lead naturally to key insights regarding bias-increasing covariates. First, we show that fixed effects for groups can act as pure bias amplifiers. This is novel because fixed effects are not instruments, which have been the focus of the bias amplification literature to date. Moreover, fixed effects are often thought to be a canonical technique for absorbing unmeasured group-level confounding, so demonstrating that they can increase bias in general runs counter to common understanding and practice. Second, we introduce the concept of bias "unmasking," which explains why even variables that do not amplify bias per se may still lead to net increased bias. In Section 3, we examine two case studies in which the causal effect is known, and where confounding is likely to be present, to estimate and decompose biases into their constituent parts. In one case study, amplification is a major contributor to net bias. In the other, the inclusion of covariates leads to a larger net bias due to "unmasking" of unobserved confounder bias. These examples reinforce prior 
advice to avoid inadvertently controlling for instruments when trying to infer causal effects from data where the causal variable was not randomized. They also illustrate why applied researchers might be concerned about arbitrary use of fixed effects for groups in non-experimental studies. In Section 4, we introduce an important addition to graphical displays for sensitivity analysis to help practitioners assess the potential for amplification and unmasking. We summarize our findings in Section 5.

\section{Bias Decomposition}

In this section, we establish the conditions under which a researcher would want to condition on a set of covariates, $X$, in estimating the effect of a treatment, $Z$, on an outcome, $Y$. To help make ideas concrete, we will map this notation to the case study presented in Section 3. Therefore, we conceptualize $Z$ as a pre recorded get-out-the-vote (GOTV) phone call and $Y$ as voter turnout.

We start in Section 2.1 with a simple case where $X$, a matrix of one or more covariates, is being considered for inclusion or exclusion in its entirety. To fix ideas, first consider $X$ to include only a randomized assignment to receive the get-out-the-vote call, which acts as an instrument (for more details, see Section 3). Then, in Section 2.2, we generalize those results to the case where we want to know whether to include all of the covariates in $X$ in the conditioning set given that some of them will be included in the conditioning set. We start with the simpler case for clarity of exposition, and the generalization allows us to apply the bias decompositions to more realistic data applications. We then provide conditions specific to models that include fixed effects for groups to demonstrate how they may act as pure bias amplifiers.

\subsection{The Case of a Single Set of Conditioning Variables}

Mathematically, describing the magnitude of bias incurred by failure to satisfy the selection on observables assumption requires additional assumptions about the relationships between variables. We derive our results using the linear model, as has been done in related work (Clarke 2005, 2009; Pearl 2010; Ding and Miratrix 2014; Steiner and Kim 2016) and which has the advantage of tying this work into more general results regarding omitted variable bias. ${ }^{1}$

To proceed, consider a linear model relating voter turnout, $Y$, with the causal variable, contact via a get-out-the-vote phone call, $Z$,

$$
Y=Z \tau+X \beta^{y}+U \zeta^{y}+\epsilon^{y}
$$

In general, $X$ could represent a matrix of observed covariates; for our motivating example, we will include only the randomized treatment assignment, which acts as an instrument. $U$ is an unobserved confounder; in this setting $U$ might represent degree of political engagement. As in Carnegie, Hill, and Harada (2014a) and Imbens (2003), we make the simplifying assumption that $U \perp X$. We can justify this assumption by conceptualizing $U$ as the portion of the political engagement that is orthogonal to the randomized treatment assignment. For the sake of clarity, and without loss of generality, we also assume that variables are mean centered.

To frame this model as a causal model, we need to explicitly incorporate potential outcomes that formalize the counterfactual possibilities for the outcome under control and treatment conditions, $Y(0)$ and $Y(1)$, respectively (Rubin 1974). These represent the voting behavior (voted or did not) if not contacted and if contacted, respectively. Thus, we write the causal version of the model as $E[Y(Z) \mid Z, X, U]=Z \tau+X \beta^{y}+U \zeta^{y}$. Although we do not posit the functional form of the unconditional relationship between $Z$ and $U$, we do not rule out dependence between these variables; in fact, if they were independent, then $U$ would not be a confounder. For instance, in our example, we expect that those who are contacted have a latent trait, a feature of their personality, that makes them more prone to answering the phone that is correlated with a willingness to vote.

\footnotetext{
${ }^{1}$ We focus on the linear model; we expect that many of the results hold, broadly speaking, for generalized linear models (GLM). However, GLM models come with a host of their own problems with respect to bias. For example, coefficients from unadjusted and covariate adjusted logistic regression models are not comparable (Freedman 2008; VanderWeele and Arahc 2011; Breen, Karlson, and Holm 2013), a problem sometimes referred to as "non-collapsibility" (cf. VanderWeele 2015). A discussion of the bias of adjusted and unadjusted GLM estimators is beyond the scope of this article.
} 
In order to identify a causal effect with this model, we need to assume ignorability holds conditional on everything in these models. Formally, this requires $\{Y(z)\}_{z \in \mathcal{Z}} \perp Z \mid X, U$, where $\mathcal{Z}$ is the set of all possible levels of the treatment. In our context, this means that among those who are in the treatment group if we subset on potential voters who are the same in terms of their willingness to vote, then whether or not contact was actually made with them is randomly assigned. For the remainder of the article, we will drop the potential outcome notation with the understanding that the potential outcomes are implicit in our modeling assumptions.

Next we utilize bias expressions to examine the cases where $X$ is omitted from the conditioning set and when it is included in the conditioning set. We compare the two biases and show how to decompose these into constituent parts. Our derivation relies on a partition of the predictor set, in which letters $S$ and $O$ stand for included ("seen") and omitted sets, respectively. See Section A of the online Supplementary Materials for further details.

First, suppose one calculates the unadjusted estimate of $\tau$ in equation (1) by simply regressing $Y$ on $Z$ (ignoring known covariates $X$ ). This translates to substituting $S=[Z]$ and $O=[X U]$ in (A.2) in the online Supplementary Materials and yields an expression for the omitted variable bias of the crude estimator:

$$
\begin{aligned}
\operatorname{Bias}\left[\hat{\tau}_{\mathrm{Y} \mid \mathrm{Z}}\right] & =\left(Z^{\prime} Z\right)^{-1} Z^{\prime} X \beta^{y}+\left(Z^{\prime} Z\right)^{-1} Z^{\prime} U \zeta^{y} \\
& =\chi+v,
\end{aligned}
$$

where $\chi \equiv\left(Z^{\prime} Z\right)^{-1} Z^{\prime} X \beta^{y}$ is the bias due to omitting $X$ and $v \equiv\left(Z^{\prime} Z\right)^{-1} Z^{\prime} U \zeta^{y}$ is the bias due to omitting $U{ }^{2}$ These make sense because the bias due to omitting $X$ is made large when $X$ is a strong predictor of the outcome (as reflected in the magnitude of $\beta^{y}$ ) conditional on the $Z$. The bias due to omitting $U$ is made large when $U$ is a strong predictor of the outcome conditional on $Z$ (as reflected in $\left.\zeta^{y}\right){ }^{3}$ The symbols $\chi$ and $v$ are used as a shorthand to signify the constituent parts of the bias, as these Greek letters most closely resemble their Latin alphabet counterparts.

To understand the impact of the randomized treatment assignment, $X$, on bias, we now calculate the bias when estimating $\tau$ in a new model that includes $X$ in the conditioning set, $S$. This translates to substituting $S=[Z X]$ and $O=[U]$ in (A.2) in the online Supplementary Materials. The bias can be written in partitioned notation as follows:

$$
\operatorname{Bias}\left[\begin{array}{c}
\hat{\tau}_{\mathrm{Y} \mid \mathrm{ZX}} \\
\hat{\beta}^{y}
\end{array}\right]=\left[\begin{array}{cc}
Z^{\prime} Z & Z^{\prime} X \\
X^{\prime} Z & X^{\prime} X
\end{array}\right]^{-1}\left[\begin{array}{c}
Z^{\prime} \\
X^{\prime}
\end{array}\right] U \zeta^{y} .
$$

Using the inverse of the partition matrix and selecting off the element that corresponds to the coefficient on the causal variable $Z$ (see Section A of the online Supplementary Materials), write

$$
\begin{aligned}
\operatorname{Bias}\left[\hat{\tau}_{Y \mid Z X}\right] & =\left(Z^{\prime} Z-Z^{\prime} X\left(X^{\prime} X\right)^{-1} X^{\prime} Z\right)^{-1} Z^{\prime} U \zeta^{y} \\
& =\left(Z^{\prime} Z-Z^{\prime} X\left(X^{\prime} X\right)^{-1} X^{\prime} Z\right)^{-1}\left(Z^{\prime} Z\right)\left(Z^{\prime} Z\right)^{-1} Z^{\prime} U \zeta^{y} \\
& =\left(Z^{\prime} Z-Z^{\prime} X\left(X^{\prime} X\right)^{-1} X^{\prime} Z\right)^{-1}\left(Z^{\prime} Z\right) v \\
& =\frac{Z^{\prime} Z}{\left(Z^{\prime} Z-Z^{\prime} H_{X} Z\right)} v=\frac{S S T^{*}}{\left(S S T^{*}-S S R^{*}\right)} v \\
& =\left(\frac{1}{1-r_{Z \mid X}^{2}}\right) v=v+\left(\frac{r_{Z \mid X}^{2}}{1-r_{Z \mid X}^{2}}\right) v \\
& =v+\alpha,
\end{aligned}
$$

\footnotetext{
${ }^{2}$ Following (Greene 2000), these expressions assume that $X$ and $Z$ and $U$ are non-stochastic. Generalizing to stochastic regressors involves taking the expectation of these expressions; these are omitted for the sake of clarity.

${ }^{3}$ In our example, this translates understanding whether the randomized treatment assignment is a stronger predictor of whether or not someone will vote than the unobserved willingness to vote characteristic.
} 
where $H_{X}=X\left(X^{\prime} X\right)^{-1} X^{\prime}$ is the hat matrix associated with the regression; since we assume $E(Z)=0$, $\mathrm{SST}^{*}=\mathrm{SST}+n E(Z)^{2}$ is the total sum of squares, and $\mathrm{SSR}^{*}=\mathrm{SSR}+n E(Z)^{2}$ is the regression sum of squares, so that SSR/SST is $r_{Z \mid X}^{2}$, or the coefficient of determination, $R$-squared, in the regression of $Z$ on $X$. Last, we make the identification $\alpha \equiv\left(\frac{r_{Z \mid X}^{2}}{1-r_{Z \mid X}^{2}}\right)$ v. The term $\left(\frac{1}{1-r_{Z \mid X}^{2}}\right)$ can be referred to as the amplification factor; importantly, this term is identified. It reflects the extent to which the covariates, in this case the assignment to receive a get-out-the-vote phone call, predict actually receiving the treatment, the phone call.

The amplification factor is particularly problematic if $X$ accounts for a great deal of variation in $Z$, as noted by Pearl (2010). So, in our example, if most of the people assigned to receive a phone call actually received the phone call, then this term would be large. The term $\alpha \equiv\left(\frac{r_{Z \mid X}^{2}}{1-r_{Z \mid X}^{2}}\right) \cup$ gives the change in bias attributable to amplification; call it the net amplification bias. See Section B in the online Supplementary Materials for intuition and context provided by another example.

A careful comparison of equations (4) and (2) reveals two key insights about adding $X$ to the conditioning set of covariates. First, note the bias term in equation (2) associated with omitting $X$, namely, $\chi \equiv\left(Z^{\prime} Z\right)^{-1} Z^{\prime} X \beta^{y}$. Again, this term is large when $X$ is highly predictive of $Y$. This term is absent in equation (4) because $X$ is adjusted for in this model. Second, the bias due to omitting $U$ is modified from $\left(Z^{\prime} Z\right)^{-1} Z^{\prime} U \zeta^{y}$ in equation (2) to become $\left(Z^{\prime} Z-Z^{\prime} X\left(X^{\prime} X\right)^{-1} X^{\prime} Z\right)^{-1} Z^{\prime} U \zeta^{y}$ in equation (4). The difference between these two terms results in the appearance of $-Z^{\prime} X\left(X^{\prime} X\right)^{-1} X^{\prime}$ $Z$ in the denominator, a term which is necessarily less than or equal to zero because it is ( -1 times) a quadratic form with positive definite matrix $\left(X^{\prime} X\right)^{-1}$ (cf. Greene 2000, Sections 2.8 and 2.8.1). This term is large if $X$, here the instrument, is highly predictive of the treatment receipt. Therefore, except when the term $-Z^{\prime} X\left(X^{\prime} X\right)^{-1} X^{\prime} Z$ is zero (i.e., $X$ is not correlated with $Z$ ), it shrinks the denominator in equation (4) relative to equation (2), resulting in amplification of the bias due to the unobserved $U$.

It is useful at this juncture to emphasize why instrumental variables (Angrist, Imbens, and Rubin 1996) have been a particular focus of attention in discussing bias amplifiers (Bhattacharya and Vogt 2007; Wooldridge 2009; Pearl 2010). When $X$ is an instrument, as in our example, $\beta^{y}=0$ by definition. Therefore, the bias due to omitting $X, \chi$, in equation (2) equals 0 and there can be no benefit due to removing $\chi$ bias when going from equation (2) to equation (4) and the only change in bias is an increase due to amplification, $\alpha$. In that sense, instruments can be referred to as pure amplifiers. When $X$ is a pure amplifier, it is necessarily true that $v+\alpha$ is larger in magnitude than $\chi+v$.

Amplification is only part of the story concerning change in bias when going from an unadjusted to adjusted estimator, however. Whether conditioning on $X$ increases or decreases the net bias depends on the magnitude of equation (2) relative to the magnitude of equation (4). Formally, a set of covariates, $X$, can be said to be net bias reducing only when

$$
\left|\left(Z^{\prime} Z\right)^{-1} Z^{\prime} U \zeta^{y}+\left(Z^{\prime} Z\right)^{-1} Z^{\prime} X \beta^{y}\right|>\left|\left(Z^{\prime} Z-Z^{\prime} X\left(X^{\prime} X\right)^{-1} X^{\prime} Z\right)^{-1} Z^{\prime} U \zeta^{y}\right| .
$$

Or, using our bias decomposition notation, $|v+\chi|>|v+\alpha|$. If $v$ (the bias due to omitting $U$ ) and $\chi$ (the bias due to omitting $X$ ) have the same sign, then this implies that for $X$ to be bias reducing, $|\chi|>|\alpha|$. When $v$ and $\chi$ have opposite signs, the requirement is $|\chi|>|2 v+\alpha|$.

Clearly, conditioning on $X$ can be net bias increasing in cases where the bias due to amplification, $\alpha$, is relatively large. This can happen, for instance, when the randomized treatment assignment is a strong predictor of actual contact via a get-out-the-vote message. However, conditioning on $X$ can be net bias increasing even when $r_{Z \mid X}^{2}=0$ (and, hence, $\alpha=0$ ) if the bias due to omitting $U$, $v$, and the bias due to omitting $X$, $\chi$, have opposite signs and $|\chi|<2|v|$. In that case, because $\chi$ has an opposite sign to $v$ but similar magnitude, it can be said to be masking (or canceling) $v$ in equation (2). In that case, the $\chi$ is a "good" bias because it cancels with $v$, rendering the net bias of the unadjusted estimator closer to zero than that of the adjusted estimator. 
That bias due to omitting a known covariate $X$ can be "good" bias (because its exclusion masks bias due to the unobserved confounder) is troubling because it implies that even when $X$ is known to be predictive of $Y$, including it in the conditioning set of covariates may increase overall bias. To know whether removing $\chi$ bias improves net bias or not, one must know something about $v$ that is not identified. In light of this observation, it is clear that none of the existing recommendations for practice provide complete guidance on whether to condition on a covariate, or a set of covariates.

\subsection{The Case of Two Sets of Conditioning Variables}

In this section, we generalize the above results to the case in which we want to decide whether to include all of the covariates in $X$ in the conditioning set given that some of them will be included in the conditioning set. Notationally, first partition the matrix of covariates such that $X=\left[X_{1} X_{2}\right]$. Now assume that $X_{2}$ will certainly be in the conditioning set and the question is whether to also include $X_{1}$ in the conditioning set. This is a very common situation. The results for bias amplification are analogous to the prior case - we simply condition on $X_{2}$ throughout the derivation. We omit unnecessary detail.

The model can now be written

$$
Y=Z \tau+X_{1} \beta_{1}^{y}+X_{2} \beta_{2}^{y}+U \zeta^{y}+\epsilon^{y},
$$

where the $U$ is independent of both $X_{1}$ and $X_{2}$. Omitting $X_{1}$ from the conditioning set leads to

$$
\operatorname{Bias}\left[\hat{\tau}_{Y \mid Z X_{2}}\right]=\chi^{*}+v^{*}
$$

where $v^{*} \equiv\left(\frac{1}{1-r_{Z \mid X_{2}}^{2}}\right) v$ likewise, $\chi^{*} \equiv\left(\frac{1}{1-r_{Z \mid X_{2}}^{2}}\right) \chi_{1}$ and $r_{Z \mid X_{2}}^{2}$ is the $R$-squared in the regression of $Z$ on $X_{2}$.

Including $X_{1}$ in the conditioning set leads to

$$
\operatorname{Bias}\left[\hat{\tau}_{\mathrm{Y} \mid \mathrm{ZX}} \mathrm{X}_{2}\right]=v^{*}+\alpha^{*} .
$$

Here the net amplification bias, $\alpha^{*} \equiv\left(\frac{r_{Z \mid X_{1} X_{2}}^{2}-r_{Z \mid X_{2}}^{2}}{1-r_{Z \mid X_{1} X_{2}}^{2}}\right) v^{*}$, is defined only slightly differently from $\alpha$ above and the amplification factor can be written $\left(\frac{1-r_{Z \mid X_{2}}^{2}}{1-r_{Z \mid X_{1} X_{2}}^{2}}\right)$. Here $r_{Z \mid X_{1} X_{2}}^{2}$ is the $R$-squared in the regression of $Z$ on $X_{1}$ and $X_{2}$.

So, conditioning on $\mathrm{X}$ is bias reducing when $\left|v^{*}+\chi^{*}\right|>\left|v^{*}+\alpha^{*}\right|$. When $v^{*}$ and $\chi^{*}$ have the same sign, the requirement is that $\left|\chi^{*}\right|>\left|\alpha^{*}\right|$. When $v^{*}$ and $\chi^{*}$ have different signs, the requirement is that $\left|\chi^{*}\right|>\left|2 v^{*}+\alpha^{*}\right|$.

\subsection{The Important Special Case of Fixed Effects}

As mentioned above, pure bias amplifiers such as instruments can be particularly problematic because there cannot be any benefit to removing $\chi$ from the bias equation since $\chi=0$. In this section, we derive the conditions in which fixed effects can be pure amplifiers - amplifying bias but providing no net improvement in bias due to removing $\chi$ bias.

To consider fixed effects under the rubric presented above, simply imagine $X$ as a matrix of indicator variables representing groups. We ignore additional covariates in this development, but the results hold for that case as well. Starting from this point of view, the term $\chi \equiv\left(Z^{\prime} Z\right)^{-1} Z^{\prime} X \beta^{y}$ in equation (2) can be written $\chi \equiv \sum_{k=1}^{K}\left(Z^{\prime} Z\right)^{-1} Z^{\prime} X_{k} \beta^{y k}$, where $X_{k}$ is the column vector from $X$ associated with the $k^{\text {th }}$ dummy variable, and $\beta^{y k}$ is the corresponding coefficient for the $k$ th group.

Now consider the case where fixed effects are pure amplifiers - when the term

$$
\chi \equiv \sum_{k=1}^{k}\left(Z^{\prime} Z\right)^{-1} Z^{\prime} X_{k} \beta^{y k}=0 \text {. Trivially, this term can be zero if the terms } \beta^{y k} \text { are all zero, that is, if }
$$

the fixed effects are instruments, but it can also be zero because the positive and negative terms sum to zero. When might those positive and negative terms net out to zero? To develop an intuition, consider a model for the treatment, $Z$, 


$$
Z=\sum_{k=1}^{K} X_{k} \beta^{z k}+U \zeta^{z}+\epsilon^{z},
$$

where $U$ is defined as above. Now make the assumption, for the sake of simplifying the exposition, that $Z$ has unit variance (in addition to having mean zero) and that the size of the $K$ groups (associated with the fixed effects) are equal and thus $E\left[X_{k}\right]=1 / K$. Then, we might write for the $k$ th dummy variable

$$
\begin{aligned}
\left(Z^{\prime} Z\right)^{-1} Z^{\prime} X_{k}= & \frac{1}{n} Z^{\prime} X_{k} \\
& =\operatorname{Cov}\left(X_{k}, Z\right)=E\left[X_{k} Z\right]-E\left[X_{k}\right] E[Z] \\
& =E\left[\left(X_{k}\right)\left(\sum_{j} X_{j} \beta^{z j}+U \zeta^{z}+\epsilon^{z}\right)\right]-E\left[X_{k}\right] E\left[\sum_{j} X_{j} \beta^{z j}+U \zeta^{z}+\epsilon^{z}\right] \\
& =E\left[\left(X_{k}\right) \sum_{j} X_{j} \beta^{z j}\right]+E\left[\left(X_{k}\right)\left(U \zeta^{z}+\epsilon^{z}\right)\right]-E\left[X_{k}\right] E\left[\sum_{j} X_{j} \beta^{z j}\right] \\
& =E\left[\left(X_{k}\right)\left(X_{k}\right) \beta^{z k}\right]-\frac{1}{K} \sum_{j} E\left[X_{j} \beta^{z j}\right] \\
& =E\left[\left(X_{k}\right)\right] \beta^{z k}-\frac{1}{K} \sum_{j} \frac{1}{K} \beta^{z j} \\
& =\frac{1}{K} \beta^{z k}-\frac{1}{K^{2}} \sum_{j=1}^{K} \beta^{z j} .
\end{aligned}
$$

We rely on $X_{k} X_{k}=X_{k}$ and $X_{k} X_{j}=0_{n}$, for $j \neq k$, as well as $E\left[X_{k} U\right]=E\left[X_{k} \epsilon^{z}\right]=0$, above. Utilizing the above, we have

$$
\begin{aligned}
\chi & \equiv \sum_{k=1}^{K}\left(Z^{\prime} Z\right)^{-1} Z^{\prime} X_{k} \beta^{y k}=\sum_{k=1}^{K} \operatorname{Cov}\left(X_{k}, Z\right) \beta^{y k} \\
& =\sum_{k=1}^{K}\left(\frac{1}{K} \beta^{z k}-\frac{1}{K^{2}} \sum_{j=1}^{K} \beta^{z j}\right) \beta^{y k} \\
& =\frac{1}{K} \sum_{k=1}^{K} \beta^{z k} \beta^{y k}-\left(\frac{1}{K} \sum_{k=1}^{K} \beta^{y k}\right)\left(\frac{1}{K} \sum_{j=1}^{K} \beta^{z j}\right) \\
& =E_{k}\left[\beta^{z k} \beta^{y k}\right]-E_{k}\left[\beta^{z j}\right] E_{k}\left[\beta^{y k}\right] \\
& =\operatorname{Cov}_{k}\left(\beta^{z k}, \beta^{y k}\right),
\end{aligned}
$$

where we use the notation $\operatorname{Cov}_{k}$ to denote that covariance is to be taken across the $K$ groups. Likewise, $E_{k}$ is expectation across the $K$ groups.

The derivation provides us with conditions in which fixed effects will be pure bias amplifiers. When $\operatorname{Cov}_{k}\left(\beta^{z k}, \beta^{y k}\right)=0$, pure amplification obtains, but clearly there should be concern when this condition is approximately met as well. One way to interpret this situation is that the group-level structure in $Y$ does not covary (or covaries extremely weakly) with the group-level structure in $Z$. In other words, the average of the products of the group effects is zero.

If the expression in the last line of equation (10) can be estimated, then the fixed effects can be avoided when they are pure bias amplifiers (or close to it). Unfortunately, the term cannot be estimated unbiasedly, or even meaningfully bounded. To see this, examine $\operatorname{Bias}\left[\hat{\beta}^{y}\right]$ in (A.5) in the online Supplementary Materials. Instead, the usual regression estimator $\beta^{y k}$ converges in probability to 


$$
\beta^{y k}-\beta^{z k}\left(Z^{\prime} Z-Z^{\prime} X\left[X^{\prime} X\right]^{-1} X^{\prime} Z\right)^{-1} Z^{\prime} U \zeta^{y} .
$$

Asymptotically, then, a quantity that one might estimate is

$$
\begin{aligned}
\operatorname{Cov}_{k}\left(\beta^{z k}, \beta^{y k}\right) & =\operatorname{Cov}_{k}\left(\beta^{z k}, \hat{\beta}^{y k}\right) \\
& =\operatorname{Cov}_{k}\left(\beta^{z k}, \beta^{y k}-\beta^{z k}\left(Z^{\prime} Z-Z^{\prime} X\left[X^{\prime} X\right]^{-1} X^{\prime} Z\right)^{-1} Z^{\prime} U \zeta^{y}\right) \\
& =\operatorname{Cov}_{k}\left(\beta^{z k}, \beta^{y k}\right)-V_{k}\left(\beta^{z k}\right)\left(Z^{\prime} Z-Z^{\prime} X\left[X^{\prime} X\right]^{-1} X^{\prime} Z\right)^{-1} Z^{\prime} U \zeta^{y} \\
& =\operatorname{Cov}_{k}\left(\beta^{z k}, \beta^{y k}\right)-V_{k}\left(\beta^{z k}\right)(v+\alpha),
\end{aligned}
$$

where $V_{k}\left(\beta^{z k}\right)$ represents the variance of the values of $\beta^{z k}$. Because $(v+\alpha)$ may take on a potentially wide range of values, equation (12) is not a useful estimator of equation (10). ${ }^{4}$

It is certainly plausible that the relationship between group effects for the treatment and outcome are unrelated, net of controls. Since this cannot be determined empirically, a researcher must take seriously the potential for this to occur. Moreover, even if fixed effects are not pure bias amplifiers, they may be bias increasing due to bias unmasking. Thus, it is quite possible that including fixed effects for groups will lead to increased absolute bias. The common rationale for including fixed effects is that they "cannot hurt; often help" is not supported by this decomposition of bias analysis. ${ }^{5}$ Moreover, emphasizing the choice between fixed and random effects as a bias versus efficiency trade-off subverts an important consideration, which is bias increasing under the inclusion of fixed effects in the presence of an unobserved confounder. While adjusting for group-level confounding, the fixed effects approach potentially introduces the two types of bias characterized in our decomposition.

\section{Case Studies}

\subsection{Case Study I: The Effect of a Get-Out-the-Vote Intervention}

In this subsection, we repurpose the data from a study of the effect of prerecorded get-out-the-vote phone calls on voter turnout (Shaw et al. 2012) to illustrate the phenomenon of bias amplification. Although the original study was a randomized experiment, we use the data to create a constructed observational study.

In the original experiment, units were assigned to a condition that received a prerecorded telephone message encouraging them to vote or to a "no message" condition. In 1597 precincts, randomization was at the precinct level. In another 5838 precincts, households were randomly assigned to treatment or control within precinct. We use the combined data file of 463,489 subjects.

Of interest in this study was the effect of contact, $Z$, on voter turnout, $Y$. However, individuals who were actually contacted may be different from individuals who were not contacted in ways that make them more likely to vote-for instance, they were less likely to have died or moved. Therefore, naively regressing turnout on contact is likely to violate the selection on observables assumption and thus yield a biased estimate of the effect of contact. Instrumental variables regression, using treatment assignment as the instrument, is the typical remedy in a situation like this with randomized assignment to treatment and strong evidence that the only pathway between the randomized assignment and the outcome is through treatment receipt. However, we are interested in illustrating bias, so we do not use instrumental variables. And instead we make comparisons between those contacted and those not contacted. Moreover, we construct a placebo test, using turnout in prior elections as outcome measures. Since we know that contact in 2006 cannot affect

\footnotetext{
${ }^{4}$ That said, in a sensitivity analysis framework, then, estimates for $\operatorname{Cov}_{k}\left(\beta^{z k}, \beta^{y k}\right)$ might be computed for posited values of the bias term in equation (4).

${ }^{5}$ In work that supports this contention, Clarke (2009) concludes that despite some awareness of the potential for bias, the common practice in political science is to include as many predictors as possible. The author does not specifically name fixed effects in the admonitions and instead uses simulation studies to characterize absolute bias differences under inclusion and exclusion of single predictors.
} 
the turnout in prior elections, the true treatment effect must be zero. Estimates that deviate from zero thus reveal the bias inherent in the estimator. ${ }^{6}$

Within the context of our constructed observational placebo study, we can test whether two types of variables act as bias amplifiers when included as covariates in the specified model. In Section 3.2, we consider treatment assignment (an instrument for contact) as a bias amplifier. In Section 3.3, we consider fixed effects for precinct as bias amplifiers. In both sections, estimates from models that include the potential bias amplifier are compared to a simple regression of turnout on contact to see which yields an estimate closer to the true parameter value of zero. If a model with the potential bias amplifier yields an estimate that is further from zero, then this is evidence that the potential bias amplifier caused a net increase in bias. Furthermore, because the causal parameter is known to be zero, the constituent components of bias $-\chi, v$ and $\alpha$-are also identified. So for each outcome, we can see if bias amplification is the cause of the net increase in bias.

\subsection{Instrument as Bias Amplifier: Analysis and Results}

Table 1, Panel A, shows the results for the analysis of the effect of conditioning on an instrument, randomized treatment assignment, on bias. ${ }^{7}$

To describe the model specification, we refer back to model equation (1). $Y$ is an $(n \times 1)$ vector of voter turnout indicators, $Z$ is an $(n \times 1)$ vector of indicators for contact, $X$ is an $(n \times 1)$ vector of indicators of treatment assignment and $U$ is the omitted confounder, assumed to have unit variance. Each row of the table conducts the analysis for a different election. The column labeled "OLS" presents the estimated coefficient on $Z$ when regressing $Y$ on $Z$ only (OLS stands for ordinary least squares). The column labeled "Inst." presents the estimated coefficient on $Z$ when regressing $Y$ on $X$ and $Z$. The column "Diff" presents the difference between the two estimates along with a bootstrapped standard error. In the columns labeled $v, \alpha$, and $\chi$, the observed bias is decomposed into constituent parts.

For the General Election 2004, the OLS estimate exhibits a bias of 0.138, whereas the model controlling for treatment is much more biased at $0.478 .^{8,9}$ The bias increase of 0.339 (an increase of $244 \%$ ) is entirely due to bias amplification, $\alpha$. That there is essentially no contribution to the bias through $\chi$ is expected given that instruments are known to be pure amplifiers. Not surprisingly, then, the unadjusted estimator is better than one that adjusts for an instrument.

Results in Table 1, Panel A, from other election years show substantively similar results. As in the case of the General Election 2004, adding the treatment indicator to the conditioning set of covariates leads to increased bias. The increase in bias is attributable to bias amplification.

Results in Table 1, Panel B, repeat this analysis for models that include additional covariates (turnout in prior elections) in the conditioning set. To describe this model specification, we refer back to model equation (5). $Y$ and $Z$ are defined as in Panel A. $X_{1}$ is the instrument, whereas $X_{2}$ is an $(n \times k)$ matrix of indicators for turnout in $k$ prior elections. For the General 2004 election outcome, $X_{2}$ included General 2002 turnout, General 2000 turnout, Primary 2004 turnout, Primary 2002 turnout, and Primary 2000 turnout. For the Primary 2004 election outcome, $X_{2}$ included General 2002 turnout, General 2000 turnout, Primary 2002 turnout, and Primary 2000 turnout. The column labeled "OLS" presents the estimated coefficient on $Z$ when regressing $Y$ on $Z$ and $X_{2}$. In the next column, labeled "Inst.," is the estimated coefficient on $Z$ when regressing $Y$ on $Z, X_{2}$, and also $X_{1}$. The remaining columns give the difference between the two estimates and the bias decomposition.

Overall, the biases are smaller in Panel B. For example, in Panel A, for General 2004 the bias due to omitting $U$, $v$, is estimated to be 0.140 , or 14 percentage points. In Panel $\mathrm{B}$, in contrast, the bias

\footnotetext{
${ }^{6}$ Another option would have been to use 2006 election turnout as the outcome and compare our observational estimates to the experimental benchmark created by the instrumental variables estimate. The downside of this approach is that this benchmark is itself noisy, making it more difficult to precisely partition the bias. We prefer using the sharp 0 of our placebo tests as a comparison.

${ }^{7}$ For the data and replication files for all tables and figures herein, see Middleton (2016).

${ }^{8}$ The standard errors are so small as to suggest that the bias is measured with great precision.

${ }^{9}$ This is a tremendous amount of bias when one considers that the outcome is a binary, $0-1$, outcome.
} 
Table 1 GOTV example with instrument as potential bias amplifier

\begin{tabular}{|c|c|c|c|c|c|c|}
\hline A. No covariates & $O L S(S E)$ & Inst. (SE) & $\operatorname{Diff}(S E)$ & $v$ & $\alpha$ & $\chi$ \\
\hline General 2004 & $\begin{array}{c}0.138 \\
(0.004)\end{array}$ & $\begin{array}{c}0.478 \\
(0.004)\end{array}$ & $\begin{array}{c}0.339 \\
(0.003)\end{array}$ & 0.140 & 0.337 & -0.002 \\
\hline General 2002 & $\begin{array}{c}0.135 \\
(0.005)\end{array}$ & $\begin{array}{c}0.451 \\
(0.004)\end{array}$ & $\begin{array}{c}0.315 \\
(0.004)\end{array}$ & 0.132 & 0.318 & 0.003 \\
\hline General 2000 & $\begin{array}{c}0.131 \\
(0.005)\end{array}$ & $\begin{array}{c}0.451 \\
(0.004)\end{array}$ & $\begin{array}{c}0.32 \\
(0.004)\end{array}$ & 0.132 & 0.318 & -0.001 \\
\hline Primary 2004 & $\begin{array}{c}0.094 \\
(0.005)\end{array}$ & $\begin{array}{c}0.285 \\
(0.004)\end{array}$ & $\begin{array}{c}0.192 \\
(0.004)\end{array}$ & 0.084 & 0.202 & 0.01 \\
\hline Primary 2002 & $\begin{array}{c}0.093 \\
(0.004)\end{array}$ & $\begin{array}{c}0.288 \\
(0.003)\end{array}$ & $\begin{array}{c}0.195 \\
(0.004)\end{array}$ & 0.085 & 0.204 & 0.009 \\
\hline Primary 2000 & $\begin{array}{c}0.113 \\
(0.004)\end{array}$ & $\begin{array}{c}0.37 \\
(0.004)\end{array}$ & $\begin{array}{c}0.257 \\
(0.003)\end{array}$ & 0.109 & 0.261 & 0.004 \\
\hline B. With covariates & $O L S(S E)$ & Inst. (SE) & Diff $(S E)$ & $v^{*}$ & $\alpha^{*}$ & $\chi^{*}$ \\
\hline General 2004 & $\begin{array}{c}0.017 \\
(0.002)\end{array}$ & $\begin{array}{c}0.077 \\
(0.002)\end{array}$ & $\begin{array}{l}0.06 \\
(0.002)\end{array}$ & 0.021 & 0.056 & -0.005 \\
\hline Primary 2004 & $\begin{array}{c}0.025 \\
(0.005)\end{array}$ & $\begin{array}{c}0.062 \\
(0.003)\end{array}$ & $\begin{array}{c}0.037 \\
(0.004)\end{array}$ & 0.017 & 0.045 & 0.008 \\
\hline
\end{tabular}

Note: Results are displayed for estimates of the effect of the get-out-the-vote intervention on a number of pretreatment outcomes, thus creating placebo tests. Column 1 reveals that linear regression results suffer from bias due to selection on unobservables. Column 2 displays results from an extension of this analysis that could exacerbate the selection bias by including the indicator for the initial randomization, which in this case acts as an instrument. The third column presents the raw difference between columns 1 and 2 . The final three columns decompose the bias into the constituent parts (see Sections 2 and 2.2).

due to omitting $U, v^{*}$, is estimated to be 0.021 or 2 percentage points. This seems to suggest that the prior vote history variables are themselves covariates that reduce bias in this example. However, we note that 2.1 percentage points is still a substantively large bias. Moreover, the ratio $\frac{\alpha^{*}}{v^{*}}$ is similar to $\frac{\alpha}{v}$ above; bias due to amplification, $\alpha^{*}$, is over $250 \%$ the size of the bias due to omitting $U, v^{*}$.

\subsection{Fixed Effects as Bias Amplifiers: Analysis and Results}

Next, consider the implications for bias when adding fixed effects for precinct to the model specification. Table 2 presents these results. In Panel A, referring back to model equation (1), $Y$ and $Z$ are defined as the turnout indicators and contact indicators, as above, whereas $X$ is now an $(n \times K)$ matrix of dummy variable indicators for the $K$ precincts.

Examining the results for 2004 election turnout, the fixed effects (FE) model is much more biased than the model without fixed effects; when regressing $Y$ on $Z$, only the estimate is 0.138 , compared with 0.272 when regressing $Y$ on $Z$ and $X$. The net increase in bias is $97 \%$. Here again, the major factor in the bias difference is bias amplification, $\alpha$. Fixed effects are essentially pure bias amplifiers, as evidenced by the fact that there is virtually no bias associated with omitting them $(\chi=0.001)$. We reiterate: group fixed effects have the potential to increase absolute bias by way of pure bias amplification.

Results in Table 2, Panel A, from other election years show substantively similar results for adding fixed effects to the model specification. Adding the fixed effects to the conditioning set of covariates leads to increased bias due to bias amplification.

Panel B of Table 2 presents the analysis where additional covariates are included in the specification. Again, refer back to model equation (5) to see the model specification. $Y$ and $Z$ are specified as in Panel A. Here, $X_{1}$ is a matrix of dummy variables for precinct and $X_{2}$ includes the prior election turnout indicators as in Table 1, Panel B.

Results again show that fixed effects have amplified bias. Although the amount of bias starts off lower for these models, the amplification factor is about the same, roughly doubling the bias of the estimate. 
Table 2 GOTV example with set of fixed effects as potential bias amplifier

\begin{tabular}{|c|c|c|c|c|c|c|}
\hline A. No covariates & $O L S(S E)$ & $F E(S E)$ & Diff $(S E)$ & $v$ & $\alpha$ & $\chi$ \\
\hline General 2004 & $\begin{array}{c}0.138 \\
(0.004)\end{array}$ & $\begin{array}{c}0.272 \\
(0.004)\end{array}$ & $\begin{array}{c}0.134 \\
(0.004)\end{array}$ & 0.137 & 0.135 & 0.001 \\
\hline General 2002 & $\begin{array}{c}0.135 \\
(0.005)\end{array}$ & $\begin{array}{c}0.257 \\
(0.004)\end{array}$ & $\begin{array}{c}0.123 \\
(0.005)\end{array}$ & 0.129 & 0.128 & 0.006 \\
\hline General 2000 & $\begin{array}{c}0.131 \\
(0.005)\end{array}$ & $\begin{array}{c}0.257 \\
(0.004)\end{array}$ & $\begin{array}{c}0.126 \\
(0.004)\end{array}$ & 0.129 & 0.128 & 0.002 \\
\hline Primary 2004 & $\begin{array}{c}0.094 \\
(0.005)\end{array}$ & $\begin{array}{c}0.172 \\
(0.003)\end{array}$ & $\begin{array}{c}0.077 \\
(0.005)\end{array}$ & 0.087 & 0.086 & 0.007 \\
\hline Primary 2002 & $\begin{array}{c}0.093 \\
(0.004)\end{array}$ & $\begin{array}{c}0.174 \\
(0.003)\end{array}$ & $\begin{array}{c}0.081 \\
(0.004)\end{array}$ & 0.087 & 0.086 & 0.006 \\
\hline Primary 2000 & $\begin{array}{c}0.113 \\
(0.004)\end{array}$ & $\begin{array}{c}0.217 \\
(0.004)\end{array}$ & $\begin{array}{c}0.104 \\
(0.003)\end{array}$ & 0.109 & 0.108 & 0.004 \\
\hline B. With covariates & $O L S(S E)$ & $F E(S E)$ & $\operatorname{Diff}(S E)$ & $v^{*}$ & $\alpha^{*}$ & $\chi^{*}$ \\
\hline General 2004 & $\begin{array}{c}0.017 \\
(0.002)\end{array}$ & $\begin{array}{c}0.037 \\
(0.001)\end{array}$ & $\begin{array}{c}0.02 \\
(0.002)\end{array}$ & 0.018 & 0.019 & -0.001 \\
\hline Primary 2004 & $\begin{array}{c}0.025 \\
(0.005)\end{array}$ & $\begin{array}{c}0.037 \\
(0.002)\end{array}$ & $\begin{array}{c}0.011 \\
(0.005)\end{array}$ & 0.018 & 0.019 & 0.007 \\
\hline
\end{tabular}

Note: The columns are otherwise similar to those in Table 1.

\subsection{Case Study II: The Effect of Selecting a Disadvantaged Village Council President}

In the previous case study, we demonstrated a situation where bias amplification resulted from either adding an instrument or adding fixed effects to the conditioning set of covariates. This amplification occurred whether or not there were additional conditioning covariates specified in the model. In this section, we consider a case study that repurposes data from another study (Dunning and Nilekani, 2013) to provide an example where bias amplification per se is not a major concern but where fixed effects nonetheless lead to a large increase in net bias, as the bias due to $v$ is "unmasked."

The original article examined the effect of having a village council president from a disadvantaged group (scheduled cast or scheduled tribe) on programmatic spending in India. In certain locations in India, council seats are reserved for disadvantaged groups on a rotating basis. Villages were assigned to have a reserved seat by first creating a list of councils within each district sorted by size of the population of the target disadvantaged group. Then, councils above a certain cutoff had their presidencies reserved for a disadvantaged group. In subsequent elections, the list was rotated so that a different set of villages had reserved seats. The original study capitalized on the list rotation scheme to conduct a quasi-experimental study that compared cities just above the cutoff to cities just below.

\subsection{Analysis and Results}

We reuse these data in a way not intended by the original study in order to induce confounding and study the resulting bias. We induce confounding by using the entire data set, not just the quasiexperimental pairs. Including data from all villages introduces confounding because villages higher on the list are not valid counterfactual cases for those further down given that they were sorted by the population of the disadvantaged groups.

Next, because outcome data exist for a time period before the assignment of the treatment, we were able once again to conduct a placebo test, whereby the effect of the treatment on the outcomes in a prior time period could be analyzed..$^{10}$ As above, since the treatment cannot affect outcomes in

\footnotetext{
${ }^{10}$ We examined the "effect" of seats reserved in the 2007 election on outcomes from 2006 . We also limited the data set to those villages that did not have a reserved presidency in 2005-2006 election years.
} 
Table 3 Village council presidency example with fixed effects

\begin{tabular}{|c|c|c|c|c|c|c|}
\hline A. No Covariates & $O L S(S E)$ & $F E(S E)$ & Diff $(S E)$ & $v$ & $\alpha$ & $\chi$ \\
\hline Ashraya & $\begin{array}{c}3.4 \\
(2.6)\end{array}$ & $\begin{array}{c}0.4 \\
(1.9)\end{array}$ & $\begin{array}{c}-3.0 \\
(2.8)\end{array}$ & 0.4 & 0.1 & 3.0 \\
\hline IAY & $\begin{array}{c}33.4 \\
(12.4)\end{array}$ & $\begin{array}{r}-34.0 \\
(8.9)\end{array}$ & $\begin{array}{r}-67.5 \\
(9.5)\end{array}$ & -30.0 & -4.1 & 63.4 \\
\hline Ambedkar & $\begin{array}{c}-0.6 \\
(0.6)\end{array}$ & $\begin{array}{c}-0.8 \\
(0.8)\end{array}$ & $\begin{array}{c}-0.2 \\
(0.2)\end{array}$ & -0.7 & -0.1 & 0.1 \\
\hline MGNREGA & $\begin{array}{c}0.5 \\
(3.5)\end{array}$ & $\begin{array}{c}-0.5 \\
(2.7)\end{array}$ & $\begin{array}{r}-1.0 \\
(1.0)\end{array}$ & -0.5 & 0.0 & 1.0 \\
\hline Water Infrastructure & $\begin{array}{c}0.3 \\
(3.9)\end{array}$ & $\begin{array}{r}-10.2 \\
(4.0)\end{array}$ & $\begin{array}{r}-10.5 \\
(2.6)\end{array}$ & -9.0 & -1.2 & 9.3 \\
\hline Latrines & $\begin{array}{r}-12.3 \\
(5.8)\end{array}$ & $\begin{array}{c}-5.7 \\
(5.3)\end{array}$ & $\begin{array}{c}6.7 \\
(4.0)\end{array}$ & -5.0 & -0.6 & -7.3 \\
\hline Community Latrines & $\begin{array}{c}0.2 \\
(0.2)\end{array}$ & $\begin{array}{c}0.1 \\
(0.1)\end{array}$ & $\begin{array}{c}-0.2 \\
(0.1)\end{array}$ & 0.0 & 0.0 & 0.2 \\
\hline B. With Covariates & $O L S(S E)$ & $F E(S E)$ & Diff $(S E)$ & $v^{*}$ & $\alpha^{*}$ & $\chi^{*}$ \\
\hline Ashraya & $\begin{array}{c}1.1 \\
(1.7)\end{array}$ & $\begin{array}{c}1.5 \\
(2.2)\end{array}$ & $\begin{array}{c}0.4 \\
(1.8)\end{array}$ & 1.3 & 0.2 & -0.3 \\
\hline IAY & $\begin{array}{c}-5.9 \\
(7.5)\end{array}$ & $\begin{array}{r}-10.0 \\
(6.8)\end{array}$ & $\begin{array}{c}-4.0 \\
(5.4)\end{array}$ & -8.9 & -1.1 & 2.9 \\
\hline Ambedkar & $\begin{array}{c}-0.8 \\
(0.8)\end{array}$ & $\begin{array}{c}-1.6 \\
(1.5)\end{array}$ & $\begin{array}{c}-0.8 \\
(0.8)\end{array}$ & -1.5 & -0.1 & 0.6 \\
\hline MGNREGA & $\begin{array}{c}-0.3 \\
(3.6)\end{array}$ & $\begin{array}{c}-0.6 \\
(3.2)\end{array}$ & $\begin{array}{c}-0.3 \\
(0.7)\end{array}$ & -0.6 & 0.0 & 0.3 \\
\hline Water Infrastructure & $\begin{array}{c}1.8 \\
(3.5)\end{array}$ & $\begin{array}{c}-4.1 \\
(2.8)\end{array}$ & $\begin{array}{c}-5.9 \\
(1.8)\end{array}$ & -3.6 & -0.4 & 5.4 \\
\hline Latrines & $\begin{array}{c}-5.1 \\
(5.0)\end{array}$ & $\begin{array}{c}-1.8 \\
(5.5)\end{array}$ & $\begin{array}{c}3.4 \\
(3.3)\end{array}$ & -1.6 & -0.2 & -3.6 \\
\hline Community Latrines & $\begin{array}{c}0.1 \\
(0.2)\end{array}$ & $\begin{array}{c}0.2 \\
(0.2)\end{array}$ & $\begin{array}{c}0.1 \\
(0.1)\end{array}$ & 0.2 & 0.0 & -0.1 \\
\hline
\end{tabular}

the past, the true value of the parameter is known to be zero. Estimates from the data can be compared to the true benchmark of zero and deviations from zero can be considered evidence of bias.

Our analysis compared models with and without fixed effects for district for each of a number of outcome measures. The estimates from the two models can be compared to see which is closer to the true parameter value of zero. If the fixed effects model is further from zero, then this is evidence that fixed effects cause a net increase in bias.

The outcome measures reflect seven government programs. Table 3 provides the names of the programs. Outcomes are measured in thousands of rupees for the first five outcomes and in number of latrines for the last two.

Table 3 presents our results. Again, referring back to the model in equation (1), $Y$ is an $(n \times 1)$ vector of expenditures (or number of latrines for the last two outcomes), $Z$ is an $(n \times 1)$ vector of indicators for treatment assignment (reserved council presidency), and $X$ is an $(n \times k)$ matrix of indicators of district (taluk). In Panel A, Table 3, the column labeled "OLS" gives the estimated coefficient on $Z$ when regressing $Y$ on $Z$ only. The column labeled "FE" gives the estimated coefficient on $Z$ when regressing $Y$ on $Z$ and $X$.

Results in Panel A of Table 3 show that in three of seven cases (Ashraya, Latrines, and Community Latrines), fixed effects appear to be moving estimates in the direction of zero. In two other cases (Indira Awaas Yojana (IAY) Scheme and Ambedkar), the estimates are moving away from zero but only slightly so. In the case of the Mahatma Ghandi National Rural Employment Guarantee Act (MGNREGA) the result is a tossup, with bias moving from 0.5 to -0.5 with the addition of the fixed effects. 
In the case of the Water Infrastructure, however, the fixed effects estimate is much further from zero compared with the unadjusted estimate (0.3 compared with -10.2). The bootstrapped standard error suggests that this is a statistically significant difference.

In the last three columns of the table, the observed biases are decomposed into constituent parts: the bias due to the unobserved confounder (v), the bias due to amplification when controlling for $X$ $(\alpha)$, and the bias due to omitting $X$ from the conditioning set $(\chi)$. In decomposing this bias in the case of Water Infrastructure, we can examine what is happening; the bias due to omitting $X$ is roughly of the same magnitude as the bias due to omitting $U$, but they have opposite signs. When neither $X$ nor $U$ are controlled for in the model, the two biases cancel. In this sense, $\chi$ can be said to be "good" bias, which is masking $v$ in the unadjusted model. Bias amplification $(\alpha)$ plays a role, albeit a smaller one, accounting for about $9 \%(-0.6 /-6.7)$ of the move away from zero when going from the unadjusted to the adjusted estimator.

Panel B of Table 3 shows the results for the model that includes several additional covariates in the conditioning set. Referring to model equation (5), $Y$ and $Z$ are defined as in Panel A. $X_{1}$ is a matrix of dummy variables for district. $X_{2}$ is an $(n \times 7)$ matrix of covariates including village expenditures for the year, village population, population of scheduled caste members, population of scheduled tribe members, size of the literate population, and size of the working population.

The values in the OLS column of Panel B, Table 3, are the estimated coefficient on $Z$ when regressing $Y$ on $Z$ and $X_{2}$. The values in the FE column are the estimated coefficient on $Z$ when regressing $Y$ on $Z, X_{1}$, and $X_{2}$.

Results confirm the main finding for Water Infrastructure. Including fixed effects in the model unmasks the bias due to $U, v^{*}$, making the total bias worse than when fixed effects are not included in the model.

Interestingly, results in Panel B also show that when controlling for these other covariates, $X_{2}$, fixed effects actually increase bias for six out of seven outcomes compared with two out of seven in Panel A. That additional covariates, $X_{2}$, can alter whether fixed effects help or hurt greatly complicates the question of what to include in the conditioning set for practitioners.

\section{A Sensitivity Analysis Framework}

For the case studies that we have examined, we have identified situations where adding an instrument or fixed effects to a set of conditioning variables increases bias. We can see this increase in bias because we have constructed these studies as placebo tests, whereby the true parameter value is known to be zero because the outcomes occurred before the treatment. However, our case studies provide little consolation to practitioners who do not know the true value of the parameter. The question we consider in this section is whether sensitivity analysis could be used to alert a practitioner to the potential for increases in bias that we have demonstrated.

Sensitivity analysis has been proposed as a way to visualize the potential for an unobserved confounder to bias results of an analysis (c.f. Rosenbaum and Rubin 1983; Imbens 2003; Clarke 2005,2009 ). These approaches posit the attributes of an unobserved confounder, $U$ (usually its association with treatment $Y$ and outcome $Z$ ), that would be sufficient (in addition to observed confounders) to satisfy the selection on observable assumption. Then, they calculate the amount of bias induced by failing to include $U$ in the conditional set. Typically a full sensitivity analysis repeats this exercise across a range of possible attributes for $U$ and the results can be visually displayed. If the estimated outcome changes very little except in the face of very extreme confounding by $U$, the results are said to be insensitive to omitted confounder bias. Similar attributes of observed covariates (e.g., their associations with treatment and outcome) can be used as benchmarks to help understand the range of plausible attribute values for "typical" covariates in that setting.

We modify for our purposes a new sensitivity analysis package available in R: treatsens (Carnegie, Hill, and Harada 2014a, 2014b). The tool takes a dual-parameter approach similar to that of Imbens (2003).

For a given combination of values of the sensitivity parameters (the coefficients on $U$ in the $Y$ and $Z$ models: $\zeta^{z}$ and $\zeta^{y}$, respectively), an estimate of the treatment effect, $\tau$, can be generated by 
first drawing candidate values of $U$, denoted $\cdot U$, from the distribution implied by the sensitivity parameters and then estimating the parameters of the model regressing $Y$ on $Z, X_{1}, X_{2}$, and $\cdot U$ using OLS. Call the estimate of the coefficient on $Z, \hat{\tau}\left(\zeta^{z}, \zeta^{y}\right)$. An average of this parameter estimate is taken across twenty draws of $\cdot U$ to reduce the uncertainty associated with the random draws from the distribution of $U$.

The algorithm proceeds by considering a range of possible values of $\zeta^{z}$ and $\zeta^{y}$ in a grid. Values of $\hat{\tau}\left(\zeta^{z}, \zeta^{y}\right)$ can be computed for each cell in the grid. The values in the grid can be represented on a plot with axes $\zeta^{z}$ and $\zeta^{y}$ and contours drawn representing constant values of $\hat{\tau}\left(\zeta^{z}, \zeta^{y}\right)$.

Note that for a given contour, all of the bias terms in our equations are identified. Therefore, we modify the sensitivity analysis currently available in the treatsens package to label each contour in the grid with the values of $v^{*}, \alpha^{*}$, and $\chi^{*}$, which are themselves implied by the values of the sensitivity parameters, $\zeta^{z}$ and $\zeta^{y}$, in addition to $\hat{\tau}\left(\zeta^{z}, \zeta^{y}\right)$. Additionally, we place a contour demarcating the area in which given fixed group effects would increase bias, rather than decreasing it. This modification allows the user to identify whether the areas of the parameter space where bias increases due to this addition represent manifestations of the unobserved variable $U$ that are plausible.

To calibrate the strength of the sensitivity parameters, we follow Imbens (2003) in plotting the coefficient estimates on the (standardized) observed covariates, $X_{2}$ in the framework of Section 2.2, in the data.

In the next section, we present a sensitivity analysis plot for the voter turnout experiment. Section $\mathrm{C}$ in the online Supplementary Materials presents a figure for village expenditures in India.

\subsection{Sensitivity Analysis of GOTV Outcomes}

The sensitivity analysis for the GOTV outcomes, in Figure 1, examines the potential for fixed effects to bias the estimates for the effect of contact on General 2004 turnout, presented in Panel B of Table 2 .

In interpreting Figure 1, consider the point $(0.1,0.05)$. It falls approximately on the line labeled tau $=-0.007$. The figure implies that if $\zeta^{z}=0.1$ and $\zeta^{y}=0.05$, then the true effect would be about -0.007 . The line also provides the decomposed bias $v=0.021, \alpha=0.023$, and $\chi=0.003$. From this, we can conclude that if $\zeta^{z}=0.1$ and $\zeta^{y}=0.05$, then the bias of the estimator without fixed effects, $v+\chi=0.024$, is smaller in magnitude than the bias when adjusting for fixed effects, $v+\alpha=0.044$. The figure also alerts us that the net amplification bias, $\alpha$, is relatively large in this case, being roughly $100 \%$ of the value of the omitted confounder bias, $v$, throughout the figure. As a helpful summary, the dashed line represents the threshold separating the region where fixed effects are bias increasing from the region in which the fixed effects are bias reducing. Above and to the right of the line, fixed effects are bias increasing; for all other values of $\zeta^{z}$ and $\zeta^{y}$, the fixed effects are bias reducing.

The plus signs in the figure represent estimated coefficients on the (standardized) covariates, $X_{2}$, from the outcome and treatment models. As in Carnegie, Hill, and Harada (2014a) and Imbens (2003) we interpret the plus signs as providing benchmarks that help the researcher assess the plausibility of an omitted confounder with similar properties. For example, the mark furthest from the origin, at about $(0.03,0.19)$, is plotting the coefficients on the (standardized) indicator of turnout in the 2000 general election. One interpretation is that it is likely that the sensitivity parameters corresponding to the omitted confounder could have properties similar to that of the indicator for turnout in the 2000 general election. Reassuringly, Figure 1 indeed would have provided a researcher with a warning to be wary of fixed effects for this data set.

\section{Discussion}

We have discussed the ways in which additional control covariates can increase bias, including bias amplification and bias unmasking. This decomposition made apparent a special case of pure bias amplification, in particular, when fixed effects amplify bias. The canonical example of a (pure) biasamplifying covariate in the literature to date has been instruments. However, we have shown that 


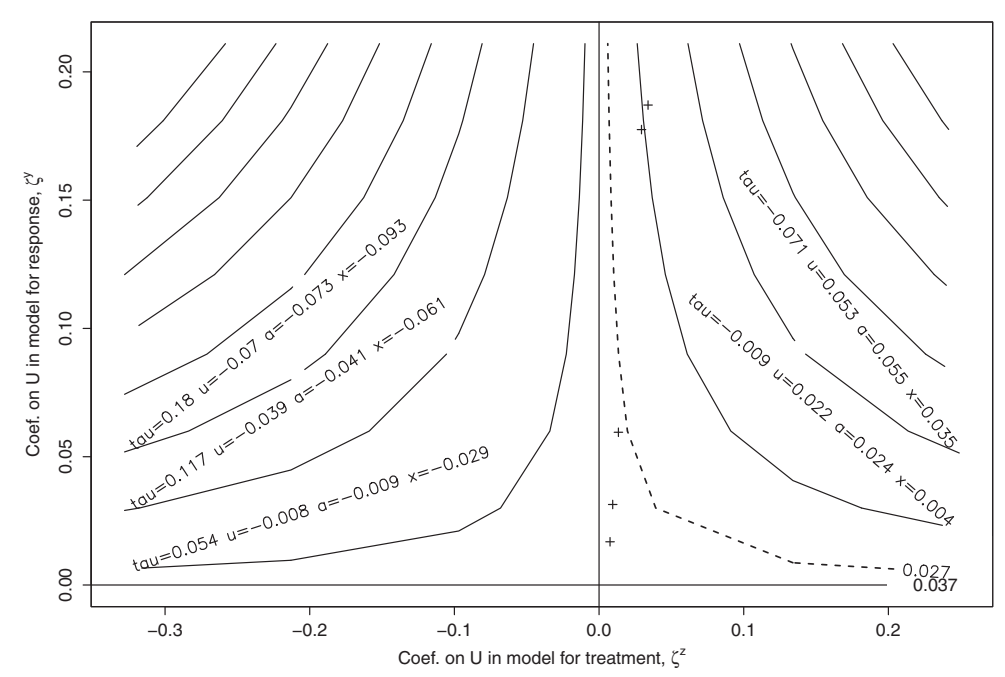

Fig. 1 Sensitivity plot for Shaw et al. (2012) data.

fixed effects can be pure bias amplifiers even though they do not act as instruments and even though they absorb heterogeneity in (and are causally related to) the outcome. Since fixed effects are often characterized as simply inefficient, rather than biased, in the econometric framework, the potential for amplification has been obscured to date. We then presented a method of visualizing the conditions under which fixed effects are bias increasing (either via unmasking or amplification) to guide the researcher via a calibrated sensitivity analysis.

Our bias decomposition delineates two scenarios in which bias increases upon inclusion of particular covariates. In the case of amplification, the bias formulas provided in this article help us better understand the circumstances under which covariates may act as bias amplifiers or bias unmaskers. Examining $\alpha \equiv\left(\frac{r_{Z \mid X}^{2}}{1-r_{Z \mid X}^{2}}\right) v$ provides some reassurance that amplification may not be a major concern in practice. It is only greater than the bias due to omitting $U$, $v$, when $r_{Z \mid X}^{2}>\frac{1}{2}$. In other words, $X$ would have to account for more than half of the variability in the assignment mechanism for amplification to have the bias to be as large as the bias due to the unobserved confounder. ${ }^{11}$ Fortunately, $r_{Z \mid X}^{2}$ is identified, a fact that should give us some idea of whether bias amplification should be a particular concern.

However, concern over the phenomenon of bias unmasking should rival concern over bias amplification. In the second case study, for example, the Water Infrastructure outcome reveals that the bias due to omitting fixed effects, $\chi$, can be large, but of opposite sign and similar magnitude compared to the bias due to the unobserved confounder, v. Omitting both the fixed effects and the unobserved confounder was preferable to adjusting for the fixed effects precisely because the two biases counterbalanced one another in the unadjusted estimate. In practice, a researcher is unlikely to know whether adjusting for covariates will unmask unobserved confounder bias. Similar observations have led to somewhat pessimistic assessment of observational analysis, for example, in Clarke (2005) and Frisell et al. (2012) (but see also Clarke [2009]).

Sensitivity analysis when considering unobserved confounders has been previously considered elsewhere (e.g., Imbens 2003; Clarke 2009; Carnegie, Hill, and Harada 2014a). We proposed an important modification to sensitivity plots aimed at increasing the information available about the potential for bias amplification. Plotting the bias decompositions $(\alpha, v$, and $\chi)$ on each of the contours should help practitioners consider bias amplification and bias unmasking.

\footnotetext{
${ }^{11}$ In the case where $X$ is a matrix of dummy variables for group, this condition is equivalent to saying that the intraclass correlation (ICC) is greater than 0.5 .
} 
Although better study designs are always the best way to address concerns over the dangers caused by failing to control for all potential confounders in observational studies, the reality is that many questions of interest are difficult or impossible to study using randomized experiments. In the absence of controlled or natural experiments, we need more tools to help applied researchers make the best choices regarding how to perform their analyses. Thoughtful consideration about the potential for bias amplification and unmasking should be part of this process. We hope that the methodology presented in this article can assist the researcher and make these ideas more concrete and fully contextualized.

\section{Funding}

This research was partially supported by Institute of Education Sciences grants R305D110037 and R305B120017.

\section{References}

Angrist, J. D., G. Imbens, and D. Rubin. 1996. Identification of causal effects using instrumental variables. Journal of the American Statistical Association 91(434):444-55.

Angrist, J. D., and J. Pischke. 2009. Mostly harmless econometrics. Princeton, NJ: Princeton University Press.

Austin, P., P. Grootendorst, and G. Anderson. 2007. A comparison of the ability of different propensity score models to balance measured variables between treated and untreated subjects: a Monte Carlo study. Statistics in Medicine 26:73453 .

Breen, R., K. Karlson, and A. Holm. 2013. Total, direct, and indirect effects in logit and probit models. Sociological Methods and Research 42(2):164191.

Brookhart, M., T. Sturmer, R. Glynn, J. Rassen, and S. Schneeweiss. 2010. Confounding control in healthcare database research. Medical Care 48:S114-20.

Bhattacharya, J., and W. Vogt. 2007. Do instrumental variables belong in propensity scores? NBER Working Paper 343 , National Bureau of Economic Research, MA.

Carnegie, N. B., J. Hill, and M. Harada. 2014a. Assessing sensitivity to unmeasured confounding using simulated potential confounders. Unpublished manuscript.

Carnegie, N.B., J. Hill, and M. Harada. 2014b. Package: TreatSens. http://www.R-project.org.

Clarke, K. A. 2005. The phantom menace. Conflict Management and Peace Science 22:341352.

2009. Return of the phantom menace. Conflict Management and Peace Science 26:46-66.

Cole, S. R., R. W. Platt, E. F. Schisterman, H. Chu, D. Westreich, D. Richardson, and C. Poole. 2010. Illustrating bias due to conditioning on a collider. International Journal of Epidemiology 39(2):417420.

D'Agostino, R. Jr. 1998. Propensity score methods for bias reduction in the comparison of treatment to non-randomized control group. Statistics in Medicine 17:314-16.

Ding, P., and L. Miratrix, 2014. To adjust or not to adjust? Sensitivity analysis of M-bias and butterfly-bias. Journal of Causal Inference 2:2-17.

Dunning, T., and J. Nilekani. 2013. Ethnic quotas and political mobilization: caste, parties, and distribution in Indian village councils. American Political Science Review 107:35-56.

Freedman, D. A. 2008. Randomization does not justify logistic regression. Statistical Science 23(2):237-49.

Frisell, T., S. Oberg, R. Kuja-Halkola, and A. Sjolander. 2012. Sibling comparison designs: bias from non-shared confounders and measurement error. Epidemiology 23(5):713-20.

Greene, W. H. 2000. Econometric analysis, 4th ed. Prentice Hall, Upper Saddle River, NJ.

Greenland, S. 2002. Quantifying biases in causal models: classical confounding vs. collider-stratification bias. Epidemiology 14:300-306.

Hill, J. 2007. Discussion of research using propensityscore matching: comments on "A critical appraisal of propensityscore matching in the medical literature between 1996 and 2003" by Peter Austin. Statistics in Medicine 27(12):2055-61.

Heckman, J., and R. Robb. 1985. Alternative methods for estimating the impact of interventions. In Longitudinal analysis of labor market data, eds. J. J. Heckman and B. Singer. Cambridge University Press, Cambridge, UK.

- 1986. Alternative methods for solving the problem of selection bias in evaluating the impact of treatments on outcomes. In Drawing inferences from self-selected samples, ed. H. Wainer. New Jersey: Lawrence Erlbaum Associates.

Imbens, G. W. 2003. Sensitivity to exogeneity assumptions in program evaluation. Recent Advances in Econometric Methodology 93(2):126-32.

Lechner, M. 2001. Identification and estimation of causal effects of multiple treatments under the conditional independence assumption. In Econometric evaluations of active labor market policies in Europe, eds. M. Lechner and F. Pfeiffer, Heidelberg: Physica.

Liu, W., M. A. Brookhart, S. Schneeweiss, X. Mi, and S. Setoguchi. 2012. Implications of M-bias in epidemiologic studies: a simulation study. American Journal of Epidemiology 176:938-48. 
Middleton, J. A. 2016. Replication data for: bias amplification and bias unmasking. http://dx.doi.org/10.791/DVN/ UO5WQ4, Harvard Dataverse.

Myers J. A., J. A. Rassen, J. J. Gagne, K. F. Huybrechts, S. Schneeweiss, K. J. Rothman, M. M. Joffe, and R. J. Glynn. 2011. Effects of adjusting for instrumental variables on bias and precision of effect estimates. American Journal of Epidemiology 174(11):1213-22.

Pearl, J. 2000. Causality. Cambridge, New York, NY.

2009. Myth, confusion, and science in causal analysis. Technical report.

2010. On a class of bias-amplifying variables that endanger effect estimates. Proceedings of UAI, pp. 417-24.

2011. Invited commentary: Understanding bias amplification. American Journal of Epidemiology 174(11):1223-27.

Rosenbaum, P. R. 2002. Observational studies. Springer, New York, NY.

Rosenbaum, P. R., and D. B. Rubin. 1983. Assessing sensitivity to an unobserved binary covariate in an observational study with binary outcome. Journal of the Royal Statistical Society Series B (Methodological) 45:212218.

Rubin, D. B. 1974. Estimating causal effects of treatments in randomized and nonrandomized studies. Journal of Educational Psychology 66:688.

- 1978. Bayesian inference for causal effects: the role of randomization. The Annals of Statistics 6(1):34-58.

. 2002. Using propensity scores to help design observational studies: application to the tobacco litigation. Health Services and Outcomes Research Methodology 2:169-88.

Shaw, D. R., D. P. Green, J. G. Gimpel, and A. S. Gerber. 2012. Do robotic calls from credible sources influence voter turnout or vote choice? Evidence from a randomized field experiment. Journal of Political Marketing 11(4):231-45.

Schisterman, E. F., S. R. Cole, and R. W. Platt. 2009. Overadjustment bias and unnecessary adjustment in epidemiologic studies. Epidemiology 20:488-95.

Steiner, P. M., and Y. Kim. 2016. The mechanisms of omitted variable bias: bias amplification and cancellation of offsetting biases. unpublished manuscript.

Sjlander, A. 2009. Propensity scores and M-structures. Statistics in Medicine 28:141620.

Sobel, M. E. 2006. What do randomized studies of housing mobility demonstrate? Causal inference in the face of interference. Journal of the American Statistical Association 101:1398-407.

Wooldridge, J. 2009. Should instrumental variables be used as matching variables? Unpublished manuscript.

Wyss, R., M. Lunt, M. A. Brookhart, R. J. Glynn, and T. Strürmer. 2014. Reducing bias amplification in the presence of unmeasured confounding through out-of-sample estimation strategies for the disease risk score. Journal of Causal Inference 2(2):131-46.

VanderWeele, T. J. 2015. Explanation in causal inference: Methods for mediation and interaction. Oxford University Press New York, NY.

VanderWeele, T. J., and O. A. Arahc. 2011. Unmeasured confounding for general outcomes, treatments, and confounders: bias formulas for sensitivity analysis. Epidemiology 22(1):4252. 